J. Appl. Numer. Optim. 3 (2021), No. 2, pp. 297-314

Available online at http://jano.biemdas.com

https://doi.org/10.23952/jano.3.2021.2.05

\title{
APPROXIMATE SOLUTIONS OF QUASI-EQUILIBRIUM PROBLEMS: LIPSCHITZ DEPENDENCE OF SOLUTIONS ON PARAMETERS
}

\author{
MOHAMED AIT MANSOUR ${ }^{1, *}$, MOHAMED AMIN BAHRAOUI $^{2}$, ADHAM EL BEKKALI $^{2}$ \\ ${ }^{1}$ Département de Physiques, LPFAS, Faculté Polydisciplinaire, Université Cadi Ayyad, Safi, Morocco \\ ${ }^{2}$ Département de Mathématiques, Faculté des Sciences et Techniques, \\ Université Abdelmalek Essaadi, Tanger, Morocco
}

\begin{abstract}
We introduce a new concept of approximate solutions to quasi-equilibrium problems (QEP) conceived as approximate fixed points of the implicit selection map associated with this problem. As an application, we consider quasivariational inequalities (QVI) for which we discuss several types of approximations from regularization perspectives to devise a consequential term of approximate solutions. Then we present sensitivity analysis results for parametric versions of (QEP) and (QVI) wherein a recent approximate version of the well-known Lim's Lemma is employed to obtain quantitative stability of approximate solutions to these problems. We finally emphasize that the proposed approximate quasi-equilibrium points converge, from both of the qualitative and quantitative stability aspects, to exact solutions. As a further consequence, we improve some previous stability results of solutions to traffic networks problems with respect to feasible flows under perturbation.
\end{abstract}

Keywords. Qausi-equilibrium problems; Quasi-variational inequalities; Equilibrium problems; Implicit selection map; Parametric perturbation.

\section{INTRODUCTION}

Let $X$ be a normed space, and let $C$ be a closed convex of $X$. Let $f: C \times C \rightarrow \mathbb{R}$ be a realvalued bifunction, and let $K: C \rightrightarrows C$ be a closed set-valued map with convex and nonempty values. In this paper, the quasi-equilibrium problem aims at finding a point $\bar{x} \in C$ such that $\bar{x} \in K(\bar{x})$ and

$$
\mathrm{QEP}(f, K(.)) \quad f(\bar{x}, y) \geqslant 0, \forall y \in K(\bar{x}) .
$$

If $K$ has a constant value $D$ with $D$ a closed convex subset of $X$, i.e., $K(x)=D$ for all $x \in C$, then $\operatorname{QEP}(f, K()$.$) collapses into the equilibrium problem \operatorname{EP}(f, D)$ in the sense of Blum and Oettli [1], which in turn encompasses many applied mathematical problems, such as, variational inequalities, Nash equilibria, fixed points and so forth. Recently, the quasi-equilibrium problem has been considered in different settings under various assumptions, for example, the existence of solutions; see, e.g., [2, 3, 4, 5, 6, 7, 8] and the references therein. For a comprehensive

\footnotetext{
${ }^{*}$ Corresponding author.

E-mail addresses: ait.mansour.mohamed@gmail.com (M. Ait Mansour), bahraoui@hotmail.com (M.A. Bahra oui), adhamcpge@gmail.com (A. El Bekkali).
}

Received July 27, 2020; Accepted April 12, 2021.

(C)2021 Journal of Applied and Numerical Optimization 
overview on equilibrium problems, we refer to $[9,10,11]$ which cover theory, algorithms and various applications.

The main focus of this paper is to establish the Lipschitz continuous dependence of solutions to $\mathrm{QEP}(f, K()$.$) with respect to parameters inherent in the constraints sets. Let us first situate$ our contribution within the previous corresponding ones in the literature. From the viewpoint of variational inequalities, Yen [12] obtained the Lipschitz continuity of solutions under parametric polyhedral constraints in Euclidean spaces on the basis of geometrical arguments namely projections that also remain valid in infinite dimensional Hilbert spaces. Later on, the stability for perturbed hemivariational inequalities was presented in [13]. Subsequently, new results on Hölder and Lipschitz estimates for solutions to $\operatorname{EP}\left(f_{p}, C_{\lambda}\right)$, where $f_{p}$ and $C_{\lambda}$ represent the perturbations of $f$ and $C$, respectively, were obtained by Ait Mansour and Riahi [14] in normed spaces by means of a non-geometrical method that considers the strong monotonicity condition. The approach proposed in [14] was adapted to parametric strong quasimonotone Stampacchia variational inequalities in [15] by considering remarkable solutions, known under star or strict solutions's terminology, that are essential in optimality conditions of quasiconvex programming problems. In concern with the qualitative stability, we refer to, for instance, Gwinner [16], and Ait Mansour and Aussel [17] in the context of variational inequalities with relevant applications.

In [18], the authors dealt with the quantitative stability of quasi-optimization problems that extend quasiconvex programming problems. In this paper, our main purpose is to extend the results of [18] to a general abstract formulation of quasi-equilibria. Such an extension would be very interesting since many applied mathematical models whose solutions are of a quasi-type, i.e., they verify a fixed point property in a parallel fulfilment of a second variational problem, however, do not fit in the programming problems such as quasi-hemivariational inequalities, quasi-variational inequalities and their related optimal control problems.

The remaining of this paper follows the organization described as follows. In the second section, we precise the notation and the basic concepts we need in our treatment. The third section is devoted to the implicit selection map $S$ of the quasi-equilibrium problem $\mathrm{QEP}(f, K()$. as defined in (3.1) below. The conditions ensuring some useful properties of $S$, such as, the non-emptiness of its domain, the convexity and the closedness features are given. In Section 4, with the help of an appropriate implicit map, we define a new concept of approximate solutions of quasi-equilibrium problems. Then, we give several possibilities that each of them enables us to simplify the presentation of these approximate solutions for the aim of a good applicability. Section 5 focuses on the transposition of our definition of approximate quasi-equilibria into quasi-variational inequalities. In particular, we provide a discussion on the meeting points with other recent approximate solutions coming from quite different regularization perspectives. Finally, in Section 6, we present our main contribution of quantitative stability of parametric versions of quasi-equilibrium problems with a special emphasis regarding the application to quasi-variational inequalities. The passage of the suggested sharp estimates from approximate solutions to exact ones is established too. We end this paper by concluding remarks and perspectives.

\section{PRELIMINARIES}

Let $X$ and $Y$ be two normed vector spaces whose norm is denoted by $\|$.$\| . For any nonempty$ subset $A$ of $X$ and any point $x \in X, d(x, A)=\inf \{\|x-y\|: y \in A\}$ stand for the distance from $x$ 
to $A$ whereas $d(x, \emptyset)=\infty$. If $B$ is another nonempty subset of $X, e(A, B)$ denotes the excess of $A$ on $B$ given by $e(A, B)=\sup \{d(a, B): a \in A\}$. We adopt the convention $e(\emptyset, A)=0$ for any subset $\emptyset \neq A \subset X$ and $e(A, \emptyset)=+\infty$. The extended Hausdorff distance between two subsets $A$ and $B$ of $X$ is given by

$$
h(A, B)=\max \{e(A, B), e(B, A)\} .
$$

Notice that the word "extended" refers to the possibility of the distance to be $\infty$. The minimal distance between two nonempty subsets $A, B$ of $X$ is denoted and given by

$$
d(A, B)=\inf \{\|x-y\|:(x, y) \in A \times B\} .
$$

When one of the sets $A$ and $B$ is empty we set $d(A, B)=h(A, B)=+\infty$. For a given map $\Phi: X \rightrightarrows X$, for every $\varepsilon>0$, we consider the notation $\varepsilon$-Fix $(\Phi):=\{x \in X: d(x, \Phi(x)) \leqslant \varepsilon\}$ to refer to the set of $\varepsilon$-approximate fixed points of $\Phi$ while we write $\operatorname{Fix}(\Phi)$ to stand for fixed points of $\Phi$, i.e., $x \in \operatorname{Fix}(\Phi)$ if and only if $x \in \Phi(x)$.

A set-valued map $F: X \rightrightarrows Y$ is said to be Lipschitz continuous relatively to a subset $D$ of its domain if there exists $\mu \geqslant 0$ such that

$$
h\left(F(x), F\left(x^{\prime}\right)\right) \leqslant \mu\left\|x-x^{\prime}\right\|, \forall x, x^{\prime} \in D .
$$

This is equivalent to

$$
F(x) \subset F\left(x^{\prime}\right)+\mu\left\|x-x^{\prime}\right\| \bar{B}, \forall x, x^{\prime} \in D,
$$

where $\bar{B}$ stands for the closed unit ball of $Y$. If $D=\operatorname{dom}(F)=X$ and (2.1) is satisfied, then $F$ is said to be globally Lipschitz continuous with constant $\mu$. If the constant in (2.1) is such that $\mu \in[0,1)$, then $F$ is said to be a contraction.

A sequence $\left(C_{n}\right)_{n}$ of sets of a normed vector space $X$ is said be convergent with respect to the Hausdorff distance to a closed set $C \subset X$ if $h\left(C_{n}, C\right)$ converges to 0 as $n$ goes to $\infty$.

We recall now basic notations and concepts of set-convergence in normed vector spaces for which we need the following collections of subsets of the set of integer numbers $\mathbb{N}$ :

$$
\mathscr{N}_{\infty}=\{N \subset \mathbb{N}: \mathbb{N} \backslash N \text { finite }\} \quad \text { and } \quad \mathscr{N}_{\infty}^{\sharp}=\{N \subset \mathbb{N}: N \text { infinite }\} .
$$

Let us give the definitions of the outer and inner limits of a sequence of subsets. Although they are valid in any topological space, we keep normed vector space for our purpose. For a sequence $\left(C_{n}\right)$ of subsets of $X$, the outer limit is the set

$$
\begin{aligned}
\limsup _{n \rightarrow+\infty} C_{n} & =\left\{x \in X: \exists N \in \mathscr{N}_{\infty}^{\sharp}, \exists x_{n} \in C_{n}(n \in N) \text { with } x_{n} \rightarrow x\right\} \\
& =\left\{x \in X: \forall V \in \mathscr{V}(x), \exists N \in \mathscr{N}_{\infty}^{\sharp}, \forall n \in N, C_{n} \cap V \neq \emptyset\right\} \\
& =\left\{x \in X: \liminf _{n \rightarrow+\infty} d\left(x, C_{n}\right)=0\right\}
\end{aligned}
$$

while the inner limit is the set

$$
\begin{aligned}
\liminf _{n \rightarrow+\infty} C_{n} & =\left\{x \in X: \exists N \in \mathscr{N}_{\infty}, \exists x_{n} \in C_{n}(n \in N) \text { with } x_{n} \rightarrow x\right\} \\
& =\left\{x \in X: \forall V \in \mathscr{V}(x), \exists N \in \mathscr{N}_{\infty}, \forall n \in N, C_{n} \cap V \neq \emptyset\right\} \\
& =\left\{x \in X: \lim _{n \rightarrow+\infty} d\left(x, C_{n}\right)=0\right\} \\
& =\left\{x \in X: \limsup _{n \rightarrow+\infty} d\left(x, C_{n}\right)=0\right\} .
\end{aligned}
$$

The limit of the sequence $\left(C_{n}\right)$ exists if the outer and inner limit sets are equal:

$$
C:=\lim _{n \rightarrow+\infty} C_{n}:=\limsup _{n \rightarrow+\infty} C_{n}=\liminf _{n \rightarrow+\infty} C_{n} .
$$


Equivalently,

$$
\limsup _{n \rightarrow+\infty} C_{n} \subset C \subset \liminf _{n \rightarrow+\infty} C_{n} .
$$

In this case, $\left(C_{n}\right)$ is said to be convergent to $C$ in the sense of Painlevé-Kuratowski convergence.

\section{Implicit SElection MAP of (QEP) PRoblems}

In this section, we define the implicit selection map, say $S$, associated with the solution map of problem $\mathrm{QEP}(f, K()$.$) and present a synthesis of its fundamental properties from the results$ on equilibrium problems available in the literature.

For any subset $D \subset C$, we denote by $\mathrm{S}(f, D)$ the set of solutions to $\operatorname{EP}(f, D)$. With this notation, we introduce the variational selection associated with the solution map of $\operatorname{QEP}(f, K()$.$) as$ follows. Let $S: C \rightrightarrows C$ be defined, for each $x \in C$, by

$$
S(x):=S(f, K(x))=\{y \in K(x): f(y, z) \geqslant 0, \forall z \in K(x)\} .
$$

It is a simple matter to observe that solutions to $\operatorname{QEP}(f, K()$.$) coincide with fixed points of S$. Then, at first, we provide conditions ensuring that the domain of $S$ in nonempty, i.e., $\operatorname{dom}(S) \neq$ $\emptyset$. This is equivalent to finding solutions to the family of equilibrium problems $\operatorname{EP}(f, K(x))_{x \in C}$ generated by the original problem. Notice that existence results and the analysis of solution sets have been investigated extensively, we refer the reader to $[7,8,19,20,21,22,23]$.

In the sequel, our synthesis considers the following conditions.

$h_{1}$ ) The map $K$ is closed and lower semicontinuous.

$h_{2}$ ) For all $x, y \in C$, for all $x_{n} \rightarrow x$ and $y_{n} \rightarrow y$, there exists a subsequence $\left(n_{k}\right)$ such that

$$
\limsup _{k} f\left(x_{n_{k}}, y_{n_{k}}\right) \geqslant 0 \Longrightarrow f(x, y) \geqslant 0 \text {. }
$$

Remark 3.1. The assumption $\left.h_{2}\right)$ is in particular satisfied if $f$ is upper semicontinuous in $(x, y)$ with respect to the strong topology of $X$, i.e., for all $x, y \in C$, for all $x_{n} \rightarrow x$ and $y_{n} \rightarrow y$,

$$
\limsup _{n} f\left(x_{n}, y_{n}\right) \leqslant f(x, y) \text {. }
$$

The following concept of the upper sign property was firstly considered by Oettli [24] and further developed by many authors, see, for example, [4].

Definition 3.1. Assume that $X$ is a normed space and $C$ is a convex subset of $X$. Let $f$ be a bifunction defined on $C \times C$. $f$ is be said to have the local upper sign property at a point $x \in C$ if there exists a convex neighborhood $V_{x}$ such that, for all $y \in V_{x}$,

$$
f\left(z_{t}, x\right) \leqslant 0, \forall t \in(0,1) \Longrightarrow f(x, y) \geqslant 0,
$$

where $z_{t}=(1-t) x+t y$. If (3.2) is satisfied for every $y \in C, f$ is be said to have the global upper sign property in $x$.

Remark 3.2. Let us emphasize that

- The upper sign property has a strong link with the pseudomonotonicity, see [5] for more details.

- The global and local upper sign property may coincide under suitable assumptions, see [4] in this regard. 
Definition 3.1 can be seen also as a particular case of [7, Definition 5.28]. It needs to establish the convexity property of the values of the selection map $S$.

Lemma 3.1. Let $f: C \times C \rightarrow \mathbb{R}$ be a bifunction. Assume that the following conditions hold:

(i) $f$ is pseudomonotone, i.e., for all $x, y \in C, f(x, y) \geqslant 0 \Longrightarrow f(y, x) \leqslant 0$;

(ii) $f$ has the upper sign property in $x$;

(iii) $f$ is quasiconvex in $y$, i.e., for any $x \in C$, for all $y, z \in C$, and any $t \in[0,1]$,

$$
f(x, t y+(1-t) z) \leqslant \max (f(x, y), f(x, z)) .
$$

Then, for every closed convex subset $D \subset C$, the set $\mathrm{S}(f, D)$ of solutions to $\operatorname{EP}(f, D)$ is convex. In particular, for any $K: C \rightrightarrows C$ set-valued map with closed and convex values, for all $x \in C$, $\mathrm{S}(f, K(x))$ is convex.

Proof Let us denote by $\mathrm{M}(f, D)$ the set of Minty equilibrium points of $f$ over $D$, i.e., $u \in$ $\mathbf{M}(f, D)$ if and only if $f(y, u) \leqslant 0$ for all $y \in D$. By the upper sign property of $f$ in $x, \mathbf{M}(f, D) \subset$ $\mathrm{S}(f, D)$ (see [4, Proposition 3.1] or [7, Lemma 5.30]). On the other hand, from the pseudomonotonicity assumption of $f$, we clearly have $\mathbf{S}(f, D) \subset \mathbf{M}(f, D)$. Hence, $\mathbf{S}(f, D)=\mathbf{M}(f, D)$. To complete the proof, it suffices to remark that the quasi-convexity of $f$ in $y$ immediately ensures that $\mathbf{M}(f, D)$ is convex.

For the existence of solutions to equilibrium problems generated by the extended quasiequilibrium formulation, the following hypotheses are considered with the same notation as in [7].

$\left(\mathbb{H}_{1}\right) f(x, x) \geqslant 0$ for all $x \in C$;

$\left(\mathbb{H}_{2}\right) F_{0}(x)=\{y \in C: f(x, y) \leqslant 0\}$ is compactly closed for all $x \in C$ i.e., $F_{0}(x) \cap B$ is closed for every compact $B \subset C$;

$\left(\mathbb{H}_{3}\right) f$ is pseudomonotone and $f(x,$.$) is quasiconvex for all x \in C$.

In addition to these assumptions, the following coercivity assumption will be needed:

$\left(\mathbb{H}_{4}\right)$ There exists a compact and convex subset $B$ of $C$ such that

$$
\forall x \in C \backslash B, \exists y \in B, f(y, x)>0 .
$$

Proposition 3.1. Let $f: C \times C \rightarrow \mathbb{R}$ be a bifunction such that either $C$ is compact or $f$ satisfies the condition $\left(\mathbb{H}_{4}\right)$. Assume that $\left(\mathbb{H}_{1}\right)$ and $h_{2}$ ) hold and the conditions (i), (ii) and (iii) of Lemma 3.1 are satisfied. Then, for every closed convex subset $D \subset C, \operatorname{EP}(f, D)$ admits a solution. In particular, for any set-valued map $K: C \rightrightarrows C$ with closed and convex values, for all $x \in C$, $\mathrm{S}(f, K(x))$ is nonempty and convex.

Proof The required conclusion will be derived from [7, Theorem 5.39] which makes appeal to the hypotheses $\left(\mathbb{H}_{1}\right),\left(\mathbb{H}_{2}\right)$ and $\left(\mathbb{H}_{3}\right)$ mentioned above. Let $D$ be any closed convex subset of $C$. Remark that $h_{2}$ ) implies the assumption $\left(\mathbb{H}_{2}\right)$, and that (i) and (iii) ensure $\left(\mathbb{H}_{3}\right)$. Then, with the condition ii) of Lemma 3.1, all of assumptions of the case (iv) in [7, Theorem 5.39] are satisfied for the problem $\operatorname{EP}(f, D)$ with $\varphi=0$ ( $\varphi$ being the bifunction defined there). Therefore, from this result, $\operatorname{EP}(f, D)$ admits a solution. In particular, for all $x \in C, S(x)=\mathrm{S}(f, K(x))$ is nonempty. The convexity of $S(x)$ comes from Lemma 3.1 for any $x \in C$.

A useful closedness property is presented as follows. 
Proposition 3.2. Assume that the conditions $\left.\left.h_{1}\right)-h_{2}\right)$ are satisfied. Then the variational selection map $S$ is closed with respect to strong topology of $X \times X$.

Proof By observing that $\left.h_{2}\right)$ implies that the set $\{(x, y) \in C \times C: f(x, y) \geqslant 0\}$ is closed, the required conclusion is immediate from [6, Proposition 2.2].

Remark 3.3. Notice that:

- Proposition 3.2 can also be proved similarly to [7, Proposition 7.1].

- In particular, it follows from Proposition 3.2 that, for every $x \in C, S(x)$ is closed.

From now on, $\mathrm{SQ}(f, K()$.$) will stand for the set of solutions to \mathrm{QEP}(f, K()$.$) , i.e., \mathrm{SQ}(f, K()$. is the set of quasi-equilibrium points of $f$ over the constraints $K($.$) . If the map K$ is a constant, i.e., for some subset $D \subset C$, for all $x \in C, K(x)=D$, then, for each $x \in C, S(x)=S(f, D)$, and in this case $\mathrm{SQ}(f, K())=.S(f, D)$.

\section{A New Concept of Approximate Solutions}

Let us introduce now the concept of approximate quasi-equilibria for problem QEP $(f, K()$. as follows.

Definition 4.1. Let $\varepsilon>0, K: C \rightrightarrows C$ a set-valued map, and $f: C \times C \rightarrow \mathbb{R}$ a bifunction. A point $u \in C$ is said to be an $\varepsilon$-approximate quasi-equilibrium point of $f$ over $K($.) (or $\varepsilon$-approximate solution to $\operatorname{QEP}(f, K()$.$) if and only if, for all \eta>0$, there exists $x \in K(u)$ such that $\|x-u\|<$ $\varepsilon+\eta$, and $x$ is a solution to $\operatorname{EP}(f, K(u))$, i.e., $x \in S(f, K(u))$.

We denote by $\varepsilon$-SQ $(f, K()$.$) the set of \varepsilon$-approximate quasi-equilibrium points of $f$ over $K(),. \varepsilon>0$.

Now, we simplify the representation of $\varepsilon-\mathrm{SQ}(f, K()$.$) under appropriate conditions, extend-$ ing [18, Proposition 3.3] from quasi-optimization problems to general quasi-equilibrium ones. Precisely, we present situations that enable us to remove $\eta$ in Definition 4.1 as follows.

Proposition 4.1. Let $K: C \rightrightarrows C$ be a closed-valued set-valued map, and let $f: C \times C \rightarrow \mathbb{R}$ be a pseudomonotone real-valued bifunction. Assume that $\left.h_{1}\right)$ and $\left.h_{2}\right)$ hold, and suppose moreover that one of the following conditions is satisfied:

a) $X$ is a reflexive Banach space;

b) The implicit selection map $S$ is compact-valued.

Then, for every $\varepsilon>0$,

$$
\varepsilon-\operatorname{SQ}(f, K(.))=\{u \in C: \exists x \in S(u):=S(f, K(u)),\|x-u\| \leqslant \varepsilon\} .
$$

Proof It is similar to the proof of the result [18, Proposition 3.3] on the stability of quasioptimization problems.

As mentioned in the introduction section, solutions to $\mathrm{QEP}(f, K()$.$) are exactly fixed points$ of implicit selection map $S$, i.e.,

$$
u \in \mathrm{SQ}(f, K(.)) \Longleftrightarrow u \in \operatorname{Fix}(S) .
$$

In the following, we underline a similar key observation for the definition of approximate quasi-equilibria. 
Remark 4.1. Under the assumptions $\left.h_{1}\right)-h_{2}$ ), and one of the assumptions $\left.a\right)-b$ ), for every $\varepsilon>0$, for a given $u \in C, u$ is an $\varepsilon$-approximate quasi-equilibrium point of $f$ over $K($.$) if and$ only if $u$ is an $\varepsilon$-fixed point of the selection map $S$, and $u$ is close to a point $x$, which is solution to $\operatorname{EP}(f, K(u))$, i.e.,

$$
u \in \varepsilon-\mathrm{SQ}(f, K(.)) \Longleftrightarrow \exists x \in \mathrm{S}(f, K(u)),\|u-x\| \leqslant \varepsilon .
$$

In particular, if the map $K$ is constant, i.e., for some subset $D \subset C$, for all $x \in C, K(x)=D$, then $\varepsilon-\mathrm{SQ}(f, K()$.$) collapses into a set of approximate equilibrium points of f$ over $D$, denoted by $\varepsilon-\mathrm{S}(f, D)$. In this case, we have

$$
u \in \varepsilon-\mathrm{S}(f, D) \Longleftrightarrow \exists x \in \mathrm{S}(f, D),\|u-x\| \leqslant \varepsilon .
$$

This implies that $d(u, S(f, D)) \leqslant \varepsilon$.

Example 4.1. Take $X=\mathbb{R}$ (the set of real numbers), $K(x)=\mathbb{R}$ for all $x \in \mathbb{R}$, and $f(x, y)=$ $y^{2}-x^{2}$. Then, $\varepsilon-\operatorname{SQ}(f, K())=.[-\varepsilon, \varepsilon]$.

From Proposition 4.1 and Remark 4.1, we immediately see that $\varepsilon-\operatorname{SQ}(f, K().) \subset \varepsilon$-Fix $(S)$. In the following, we obtain the equality in a more general setting.

Lemma 4.1. Under the assumptions $\left.\left.h_{1}\right)-h_{2}\right)$, for every $\varepsilon>0$,

$$
\varepsilon-\operatorname{Fix}(S)=\varepsilon-\operatorname{SQ}(f, K(.)) \text {. }
$$

Proof Let $\varepsilon>0$ and $u \in C$. Then, using Definition 4.1, we see that

$$
\begin{aligned}
u \in \varepsilon-\operatorname{Fix}(S) & \Longleftrightarrow d(u, S(u)) \leqslant \varepsilon \\
& \Longleftrightarrow \forall \eta>0, \exists x \in S(u),\|x-u\|<\eta+\varepsilon \\
& \Longleftrightarrow \forall \eta>0, \exists x \in S(f, K(u)),\|x-u\|<\eta+\varepsilon \\
& \Longleftrightarrow u \in \varepsilon-\operatorname{SQ}(f, K(.)) .
\end{aligned}
$$

This completes the proof.

Remark 4.2. Observe that in Lemma 4.1 and Proposition 4.1 the assumption $h_{2}$ ) can be replaced by the closedness of the set $\{(x, y) \in C \times C: f(x, y) \geqslant 0\}$.

\section{ApProximate Solutions to QUASI-VARIATIONAL INEQUALITIES}

Let us denote by $X^{\star}$ the topological dual of $X$ whose norm is denoted by $\|\cdot\|$ as the one of $X$. Let $T: C \rightrightarrows X^{\star}$ be a weakly*-compact and convex-valued set-valued map, and let $K: C \rightrightarrows C$ be a set-valued map with closed and convex values. To these objects, we associate the following quasi-variational inequality problem: Find $x \in K(x)$ such that there exists $x^{\star} \in T(x)$ and

$$
\mathrm{QVI}(T, K(.)) \quad\left\langle x^{\star}, y-x\right\rangle \geqslant 0, \forall y \in K(x) .
$$

If for some $D \subset C, K(x)=D$ for all $x \in C$, then $\mathrm{QVI}(T, K()$.$) coincides with the variational$ inequality problem $\operatorname{VI}(T, D)$ : Find $x \in D$ such that there exists $x^{\star} \in T(x)$ and

$$
\operatorname{VI}(T, D) \quad\left\langle x^{\star}, y-x\right\rangle \geqslant 0, \quad \forall y \in D .
$$

To link problem $\mathrm{QVI}(T, K()$.$) to the general formulation \mathrm{QEP}(f, K()$.$) , let us introduce the$ associated bifunction to operator $T$, denoted by $f_{T}$ and defined by

$$
f_{T}(x, y)=\sup _{x^{\star} \in T(x)}\left\langle x^{\star}, y-x\right\rangle .
$$


From [7, Lemma 8.13] the problems $\mathrm{QVI}(T, K)$ and $\operatorname{QEP}\left(f_{T}, K().\right)$ are equivalent (in the sense that they have the same set of solutions). In this case, the variational selection map is expressed as follows: $S_{T}: C \rightrightarrows C$ are defined, for each $x \in C$, by

$$
S_{T}(x):=\left\{y \in K(x): \exists y^{\star} \in T(y),\left\langle y^{\star}, z-y\right\rangle \geqslant 0, \forall z \in K(x)\right\} .
$$

This leads to the following definition.

Definition 5.1. A point $u \in C$ is said to be an $\varepsilon$-approximate solution to $\mathrm{QVI}(T, K()$.$) if and only$ if $u$ is an $\varepsilon$-approximate quasi-equilibrium point of $f_{T}$ over $K($.$) (i.e., \varepsilon$-approximate solution to $\mathrm{QEP}\left(f_{T}, K().\right)$ in the sense of Definition 4.1). Equivalently, for all $\eta>0$, there exists $x \in K(u)$, $\|x-u\|<\varepsilon+\eta$ and $x$ is a solution to $\operatorname{VI}(T, K(u))$.

Standard approximate solutions to QVI $(T, K()$.$) are defined as follows as usual.$

Definition 5.2. We say that a point $\bar{x} \in C$ is a standard approximate $\varepsilon$-solution (or simply standard $\varepsilon$-solution) to $\mathrm{QVI}(T, K()$.$) if and only if \bar{x} \in \varepsilon$-Fix $(K)$ and there exists $x^{\star} \in T(\bar{x})$ such that

$$
\left\langle x^{\star}, y-\bar{x}\right\rangle \geqslant-\varepsilon, \forall y \in K(\bar{x}) .
$$

For a discussion with the recent results on approximate solutions to $\mathrm{QVI}(T, K()$.$) introduced$ in [25] in the context of regularization techniques, we recall the following.

Definition 5.3 ([25, Definition 1.2]). Let $\varepsilon>0$.

- We say that a point $\bar{x} \in C$ is a strong $\varepsilon$-approximate solution to $\mathrm{QVI}(T, K()$.$) if and only if$ $\bar{x} \in \operatorname{Fix}(K)$ and there exists $x^{\star} \in T(\bar{x})$ such that

$$
\left\langle x^{\star}, y-\bar{x}\right\rangle \geqslant-\varepsilon\|y-\bar{x}\|, \forall y \in K(\bar{x}) .
$$

- We say that a point $\bar{x} \in C$ is a weak $\varepsilon$-approximate solution to $\mathrm{QVI}(T, K()$.$) if and only if$ $\bar{x} \in \varepsilon-\operatorname{Fix}(K)$ and there exists $x^{\star} \in T(\bar{x})$ such that

$$
\left\langle x^{\star}, y-\bar{x}\right\rangle \geqslant-\varepsilon\left\|x^{\star}\right\|, \forall y \in K(\bar{x}) .
$$

Further, we give the following definition.

Definition 5.4. We say that a point $\bar{x} \in C$ is a mixed $\varepsilon$-approximate solution to $\mathrm{QVI}(T, K()$.$) if$ and only if $\bar{x} \in \varepsilon$-Fix $(K)$ such that $\exists x^{\star} \in T(\bar{x})$ and

$$
\left\langle x^{\star}, y-\bar{x}\right\rangle \geqslant-\varepsilon-\varepsilon\left(\left\|x^{\star}\right\|+\|y-\bar{x}\|\right), \quad \forall y \in K(\bar{x}) .
$$

Of course, if $T$ is single-valued, then (5.5) becomes

$$
\langle T(\bar{x}), y-\bar{x}\rangle \geqslant-\varepsilon-\varepsilon(\|T(\bar{x})\|+\|y-\bar{x}\|), \quad \forall y \in K(\bar{x}) .
$$

Remark 5.1. Observe that

- The terminology "mixed" in Definition 5.4 is justified by the fact that the right hand of (5.5) in Definition 5.4 mixes the corresponding ones in (5.2), (5.3) and (5.4) of Definitions 5.2 and 5.3, respectively.

- Following Definition 5.3, a point $\bar{x} \in C$ is a strong $\varepsilon$-approximate solution to QVI $(T, K()$.$) ,$ for some $\varepsilon>0$ if and only if $\bar{x} \in \operatorname{Fix}(K)$ and there exists $x^{\star} \in T(\bar{x})$ such that

$$
-x^{\star} \in s-N_{K(\bar{x})}^{\varepsilon}(\bar{x})
$$


where the notation $s-N_{K(\bar{x})}^{\varepsilon}(\bar{x})$ stands for the strong $\varepsilon$-approximate normal cone to $K(\bar{x})$ at $\bar{x}$, which is given in [26, p.6] by

$$
s-N_{K(\bar{x})}^{\varepsilon}(\bar{x})=\left\{x^{\star} \in X^{\star}:\left\langle x^{\star}, x-\bar{x}\right\rangle \leqslant \varepsilon\|x-\bar{x}\|, \forall x \in K(\bar{x})\right\} .
$$

This kind of strong approximation was obtained in [25, Definition 1.1] as a result of the regularization of operator $T$.

- Following Definition 5.3, a point $\bar{x} \in C$ is a weak $\varepsilon$-approximate solution to QVI $(T, K()$.$) ,$ for some $\varepsilon>0$ if and only if $\bar{x} \in \varepsilon$-Fix $(K)$ and there exists $x^{\star} \in T(\bar{x})$ such that

$$
-x^{\star} \in w-N_{K(\bar{x})}^{\varepsilon}(\bar{x}),
$$

where the notation $w-N_{K(\bar{x})}^{\varepsilon}(\bar{x})$ denotes the weak $\varepsilon$-approximate normal cone to $K(\bar{x})$ at $\bar{x}$, which was introduced in [25, Definition 1.1] as

$$
w-N_{K(\bar{x})}^{\varepsilon}(\bar{x})=\left\{x^{\star} \in X^{\star}:\left\langle x^{\star}, x-\bar{x}\right\rangle \leqslant \varepsilon\left\|x^{\star}\right\|, \forall x \in K(\bar{x})\right\} .
$$

We emphasize that this format of weak approximation is actually suggested by similar regularization of the selection map $S$ corresponding to (QVI) (see [25] for more details).

Let us state and prove a link between approximate solutions and mixed approximate ones for problem QVI $(T, K()$.$) as follows.$

Proposition 5.1. Assume that the map $K$ satisfies $\left.h_{1}\right), C$ is a compact subset of $X$, and $T$ is Lipschitz continuous with constant $\mu \leqslant 1$. Assume that the following regularity condition hold: For all $x, y \in C$, for all $x_{n} \rightarrow x$, for all $y_{n} \rightarrow y$,

$$
\liminf _{n \rightarrow+\infty} \sup _{x_{n}^{\star} \in T\left(x_{n}\right)}\left\langle x_{n}^{\star}, y_{n}-x_{n}\right\rangle \geqslant 0 \Longrightarrow \sup _{x^{\star} \in T(x)}\left\langle x^{\star}, y-x\right\rangle \geqslant 0 .
$$

Then, for any $\varepsilon>0$, a $\varepsilon$-approximate solution to $\mathrm{QVI}(T, K()$.$) is a mixed \varepsilon$-approximate solution to $\mathrm{QVI}(T, K()$.$) .$

Proof Observe that the assumption (5.7) implies the condition $h_{2}$ ) of Proposition 4.1. Let $\varepsilon>0$. In view of Lemma 4.1, a point $\bar{x} \in C$ is an $\varepsilon$-approximate solution to $\mathrm{QVI}(T, K()$.$) if and only if$ $\bar{x} \in \varepsilon$-Fix $\left(S_{T}\right)$. Accordingly, from the compactness of $C$ and assertion (b) of Proposition 4.1, we infer the existence of a point $u \in S_{T}(\bar{x})$ such that $\|u-\bar{x}\| \leqslant \varepsilon$. This means that $d\left(\bar{x}, S_{T}(\bar{x})\right) \leqslant \varepsilon$. From $S_{T}(\bar{x}) \subset K(\bar{x})$, we have that $d(\bar{x}, K(\bar{x})) \leqslant \varepsilon$, which implies that $\bar{x} \in \varepsilon$-Fix $(K)$. In addition,

$$
\exists u^{\star} \in T(u),\left\langle u^{\star}, y-u\right\rangle \geqslant 0, \forall y \in K(\bar{x}) .
$$

Now, denote by $\bar{B}$ the closed unit ball of $X$, and write, for some $e \in \bar{B}, \bar{x}-u=\varepsilon e$.

Consequently,

$$
\left\langle u^{\star}, y-\bar{x}+\varepsilon e\right\rangle \geqslant 0, \forall y \in K(\bar{x}) .
$$

Therefore,

$$
\left\langle u^{\star}, y-\bar{x}\right\rangle \geqslant-\varepsilon\left\langle u^{\star}, e\right\rangle \geqslant-\varepsilon\left\|u^{\star}\right\|\|e\| \geqslant-\varepsilon\left\|u^{\star}\right\|, \quad \forall y \in K(\bar{x}) .
$$

Thus, since $T$ is Lipschitz continuous with constant $\mu \leqslant 1$, then

$$
\begin{aligned}
T(u) & \subset T(\bar{x})+\mu\|u-x\| B^{\star} \\
& \subset T(\bar{x})+\varepsilon B^{\star} \quad(\text { since }\|u-\bar{x}\| \leq \varepsilon),
\end{aligned}
$$


where $B^{\star}$ stands for the closed unit ball of $X^{\star}$. Hence, there exists some $x^{\star} \in T(\bar{x})$ and some $e^{\star} \in X^{\star}$ with $\left\|e^{\star}\right\| \leqslant 1$ such that $u^{\star}=x^{\star}+\varepsilon e^{\star}$. This, with the last inequality, implies that

$$
\left\langle x^{\star}+\varepsilon e^{\star}, y-\bar{x}\right\rangle \geqslant-\varepsilon\left\|u^{\star}\right\|, \forall y \in K(\bar{x}) .
$$

It follows that

$$
\left\langle x^{\star}, y-\bar{x}\right\rangle \geqslant-\varepsilon-\varepsilon\left(\left\|x^{\star}\right\|+\|y-\bar{x}\|\right), \quad \forall y \in K(\bar{x}) .
$$

\section{Parametric Versions of (QEP) : Sensitivity Analysis Results}

This section deals with the main goal of the paper, that is, the stability under parametric perturbations on the constraints for problem QEP. Precisely, given another normed space $\Lambda$ whose norm is denoted again by $\|$.$\| , we consider that the set-valued map of constraints is$ subject to a perturbation by a parameter $\lambda \in \Lambda$ as follows: $K: C \times \Lambda \rightrightarrows C,(u, \lambda) \mapsto K_{\lambda}(u):=$ $K(u, \lambda)$, assumed to have nonempty, closed and convex values. Thus, for a given bifunction $f: C \times C \rightarrow \mathbb{R}$, the suggested parametric version of $\operatorname{QEP}\left(f, K_{\lambda}().\right)$ is to find, for every $\lambda \in \Lambda$, a point $\bar{u}_{\lambda} \in K_{\lambda}\left(\bar{u}_{\lambda}\right)$ and

$$
\operatorname{QEP}\left(f, K_{\lambda}(.)\right) \quad f\left(\bar{u}_{\lambda}, y\right) \geqslant 0, \forall y \in K_{\lambda}\left(\bar{u}_{\lambda}\right) .
$$

The variational selection corresponding to $\operatorname{QEP}\left(f, K_{\lambda}().\right)$ is $S: C \times \Lambda \rightrightarrows C$ defined, for each $u \in C$ and each $\lambda \in \Lambda$, by

$$
S_{\lambda}(u):=S(u, \lambda)=\left\{y \in K_{\lambda}(u): f(y, z) \geqslant 0, \forall z \in K_{\lambda}(u)\right\} .
$$

Observe that $S_{\lambda}(u)$ represents the set of solutions to equilibrium problem $\operatorname{EP}\left(f, K_{\lambda}(u)\right), u \in$ $C$. Of course, as for the original quasi-equilibrium problem, solutions to $\mathrm{QEP}\left(f, K_{\lambda}().\right)$ are determined by fixed points of $S_{\lambda}$. In what follows, the bifunction $f$ will be supposed to verify conditions of Proposition 3.1 so that $S_{\lambda}(u)$ could be nonempty for every $\lambda \in \Lambda$ and every $u \in C$. The convexity and closedness will be automatically satisfied since $S_{\lambda}$ will be a singlevalued map under an appropriate strong monotonicity condition. Our next result establishes the contracting character of the selection map $S$, which will be fundamental in our stability analysis around a reference value $\bar{\lambda}$ of the parameter $\lambda$.

Our assumptions of this part are as follows: There exists a neighborhood $\bar{V}$ of $\bar{\lambda}$ such that the following are satisfied:

$\left(H_{0}\right) K_{\lambda}$ satisfies the condition $\left.h_{1}\right)$ for all $\lambda \in \bar{V}$ and for some constant $L \in(0,1)$ and every $u \in C$,

$$
K_{\lambda}(u) \subset K_{\lambda^{\prime}}(u)+L\left\|\lambda-\lambda^{\prime}\right\| \bar{B}, \forall \lambda, \lambda^{\prime} \in \bar{V},
$$

where $\bar{B}$ is the closed unit ball of $X$ as before.

$\left(H_{1}\right)$ Strong monotonicity-like condition: for some $m>0$,

$$
f(x, y)+f(y, x) \leqslant-m\|y-x\|, \forall x, y \in C .
$$

$\left(H_{2}\right)$ For some constant $R>0$, for all $x, y, y^{\prime} \in C$,

$$
\left|f(x, y)-f\left(x, y^{\prime}\right)\right| \leqslant R\left\|y-y^{\prime}\right\| .
$$

Remark 6.1. Notice that the assumption $\left(H_{1}\right)$ implies the condition (i) of Lemma 3.1 (the property of pseudomonotonicity). 
Lemma 6.1. Assume that either $C$ is compact or $f$ satisfies the coercivity condition $\left(\mathbb{H}_{4}\right)$ and suppose moreover that $\left.\left(H_{0}\right)-\left(H_{2}\right), h_{2}\right),\left(\mathbb{H}_{1}\right)$ and conditions (ii) and (iii) of Lemma 3.1 are satisfied. Then, for every $\lambda \in \bar{V}$, for all $u \in C$, problem $\operatorname{EP}\left(f, K_{\lambda}(u)\right)$ admits a unique solution, say $u_{\lambda}$, and moreover there exists a constant $k>0$ such that, for all $\lambda, \lambda^{\prime} \in \bar{V}$,

$$
\left\|u_{\lambda}-u_{\lambda^{\prime}}\right\| \leqslant k\left\|\lambda-\lambda^{\prime}\right\|
$$

where $k=\frac{2 R L}{m}$.

Proof Let $\lambda \in \Lambda$ and $u \in C$. Take into account the first part of the assertion in Remark 6.1 and infer from Proposition 3.1 that $\operatorname{EP}\left(f, K_{\lambda}(u)\right)$ admits a solution $u_{\lambda}$, which must be unique in view of $\left(H_{1}\right)$. The estimate (6.2) is an immediate consequence of [14, Theorem 2.2.1, Step II].

Remark 6.2. Lemma 6.1 can not be directly applied to optimization problems because of the loss of strong monotonicity condition. Indeed, for a real-valued function $h$, clearly the choice of the bifunction given by $f(x, y)=h(y)-h(x)$ is not adequate for the stability purpose in (6.2) since this bifunction does not satisfy the strong monotonicity condition $\left(H_{0}\right)$. The alternative in convex optimization is to consider the bifunction $f_{\partial h}$ defined by

$$
f_{\partial h}(x, y)=\sup _{x^{\star} \in \partial h(x)}\left\langle x^{\star}, y-x\right\rangle .
$$

where $\partial h$ is the subdifferential of $h$ in the sense of convex analysis. In this second case, it is enough to consider the strong convexity-like on $h$ to ensure $\left(H_{0}\right)$ for $f_{\partial h}$. A further alternative may be envisaged in quasi-convex programming by using characterizations via normal operators as in [15]. For the more general quasi-optimization problems, penalization techniques seem to be a good way to establish quantitative stability as shown in [18].

Remark 6.3. Since, in Lemma 6.1, the conditions $\left.h_{1}\right)-h_{2}$ ) of Lemma 4.1 are satisfied, then, for every $\lambda \in \Lambda$ and every $\varepsilon>0$, we derive that

$$
\varepsilon-\operatorname{Fix}\left(S_{\lambda}\right)=\varepsilon-\operatorname{SQ}\left(f, K_{\lambda}(.)\right) .
$$

The conclusion of Lemma 6.1 ensures, for every $\lambda \in \bar{V}$, that $S_{\lambda}$ is a single-valued map, i.e., for all $u \in C, S_{\lambda}(u)=\left\{u_{\lambda}\right\}$. Then the conditions of the case $\left.b\right)$ in Proposition 4.1 hold. Thus, for every $\varepsilon>0$,

$$
\begin{aligned}
\varepsilon-\mathrm{SQ}\left(f, K_{\lambda}(.)\right) & =\varepsilon-\operatorname{Fix}\left(S_{\lambda}\right)=\left\{u \in C: \exists x \in S_{\lambda}(u):=S\left(f, K_{\lambda}(u)\right),\|x-u\| \leqslant \varepsilon\right\} \\
& =\left\{u \in C:\left\|u-u_{\lambda}\right\| \leqslant \varepsilon\right\} .
\end{aligned}
$$

Remark 6.4. The strong monotonicity-like property in condition $\left(H_{1}\right)$ corresponds in the literature to the so-called $\varphi$-strong monotonicity in the sense of [7, Definition 3.1] with $\varphi(x, y)=$ $\|y-x\|$. Let us give an important example of this assumption. Let $T: C \rightrightarrows X^{\star}$ be an $\alpha$-strongly monotone-like operator, that is, for all $x, y \in C$, for all $x^{\star} \in T(x)$ and $y^{\star} \in T(y)$,

$$
\left\langle y^{\star}-x^{\star}, y-x\right\rangle \geqslant \alpha\|x-y\| .
$$

In this case, the bifunction $f_{T}$ defined by

$$
f_{T}(x, y)=\sup _{x^{\star} \in T(x)}\left\langle x^{\star}, y-x\right\rangle
$$


verifies $\left(H_{1}\right)$ with $m=\alpha$. Also, notice that if $T$ is bounded, i.e., there exists $R>0$ such that, for all $x \in C$, for all $x^{\star} \in T(x),\left\|x^{\star}\right\| \leqslant R$, then $f_{T}$ also satisfies $\left(H_{2}\right)$.

In the sequel we need the following extension of the condition $\left(H_{0}\right)$ :

$\left(\tilde{H}_{0}\right)$ : for some constant $L \in(0,1)$ and every $u, v \in C$,

$$
K_{\lambda}(u) \subset K_{\lambda^{\prime}}(v)+L\left(\left\|\lambda-\lambda^{\prime}\right\|+\|u-v\|\right) \bar{B}, \text { for all } \lambda, \lambda^{\prime} \in \bar{V} .
$$

Remark 6.5. A practical example of our assumption $\left(\tilde{H}_{0}\right)$ is the condition (4.8) of [9, Theorem 4.3.1] used for the algorithmic purpose of fixed points and quasi-equilibria.

Now, from Lemma 6.1, we derive the following Lipschitz stability result, which will be used for the purpose of applications.

Proposition 6.1. Replace in Lemma 6.1 the assumption $\left(H_{0}\right)$ by $\left(\tilde{H}_{0}\right)$ and suppose moreover the control condition $R<\frac{m}{2 L}$. Then, the variational selection $S$, related to the quasiequilibrium problems $\operatorname{QEP}\left(f, K_{\lambda}(.)\right)_{\lambda \in \bar{V}}$, is single-valued, that is, for every $\lambda \in \bar{V}$ and every $u \in C, S(\lambda, u)=S_{\lambda}(u)$ is reduced to a singleton $\left\{u_{\lambda}\right\}$. In addition, $S$ is a contracting mapping in $(\lambda, u)$ with constant $k:=\frac{2 R L}{m}<1$, i.e., for all $\lambda, \lambda^{\prime} \in \bar{V}$, for all $u, u^{\prime} \in C$,

$$
\left\|u_{\lambda}-u_{\lambda^{\prime}}^{\prime}\right\| \leqslant k\left(\left\|\lambda-\lambda^{\prime}\right\|+\left\|u-u^{\prime}\right\|\right) .
$$

Proof It suffice to consider a new parameter space $\tilde{M}=M \times C$ equipped with the norm $\|(\lambda, u)\|=\|\lambda\|+\|u\|$ and apply Lemma 6.1.

Remark 6.6. The conclusion of Proposition 6.1 holds true if we replace the strong monotonicitylike of $f$ in $\left(H_{1}\right)$ with the strong quasiconvexity-like of $f$ in $y$, that is, for some $\alpha>0$, for all $x \in C$, for any $y, y^{\prime} \in C$ and any $t \in[0,1]$,

$$
f\left(x, t y+(1-t) y^{\prime}\right) \leqslant \max \left(f(x, y), f\left(x, y^{\prime}\right)\right)-\alpha \min \{t, 1-t\}\left\|y^{\prime}-y\right\| .
$$

In this case, we only require standard pseudo-monotonicity (or even quasimonotonicity) on $f$, wherein, by extending the result of [27, Theorem 4.13] with a slight technical change. It is possible to get a similar estimate to (6.5) as follows. For all $\lambda \in \bar{V}$, and all $u \in C$, the set of solutions to equilibrium problem $\operatorname{EP}\left(f, K_{\lambda}(u)\right)$ reduces to a singleton $\left\{u_{\lambda}\right\}=S_{\lambda}(u)$, and moreover for some $\tau \in\left(0, \frac{1}{4}\right)$ such that $\alpha \tau<L R$ there exists a neighborhood $V$ of $\bar{\lambda}$ such that, for all $u, u^{\prime} \in C$,

$$
\left\|u_{\lambda}-u_{\lambda^{\prime}}^{\prime}\right\| \leqslant \rho_{\tau}\left(\left\|\lambda-\lambda^{\prime}\right\|+\left\|u-u^{\prime}\right\|\right), \quad \forall \lambda, \lambda^{\prime} \in V
$$

where, $\rho_{\tau}=\left(\frac{L R}{\alpha \tau}\right)+L$.

To continue the treatment for (QVI) problems, we recall the following.

Definition 6.1 ([28], see also [7]). An operator $T: C \rightrightarrows X^{\star}$ is said to have the (global) upper sign continuity at a point $x \in C$ if, for every $y \in C$, with $z_{t}=(1-t) x+t y$ for $t \in(0,1)$, the following implication holds true:

$$
\inf _{z_{t}^{\star} \in T\left(z_{t}\right)}\left\langle z_{t}^{\star}, y-x\right\rangle \geqslant 0, \forall t \in(0,1) \Longrightarrow \sup _{x^{\star} \in T(x)}\left\langle x^{\star}, y-x\right\rangle \geqslant 0 .
$$

Next, we obtain a useful consequence of Proposition 6.1 concerning the contracting property of the variational selection related to quasi-variational inequalities. 
Corollary 6.1. Assume that $\left(\tilde{H}_{0}\right)$ holds and let $T: C \rightrightarrows X^{\star}$ be a set-valued map with weakly*compact and convex values such that

$\left.c_{1}\right) T$ is bounded with constant $R>0$;

$\left.c_{2}\right) T$ is globally upper sign continuous at any point $x \in C$;

$\left.c_{3}\right) T$ is $m$-strongly monotone-like with $m>2 R L$;

$\left.c_{4}\right) T$ satisfies the regularity assumption (5.7), i.e., for all $x, y \in C$, for all $x_{n} \rightarrow x$, for all $y_{n} \rightarrow y$,

$$
\limsup _{n \rightarrow+\infty} \sup _{x_{n}^{\star} \in T\left(x_{n}\right)}\left\langle x_{n}^{\star}, y_{n}-x_{n}\right\rangle \geqslant 0 \Longrightarrow \sup _{x^{\star} \in T(x)}\left\langle x^{\star}, y-x\right\rangle \geqslant 0 .
$$

Then, the variational selection $S$ related to quasi-variational inequalities $\mathrm{QVI}\left(T, K_{\lambda}(.)\right)_{\lambda \in \bar{V}}$ is a single-valued contracting mapping in $(\lambda, u)$ with constant $k=\frac{2 R L}{m}<1$.

Proof By taking into account $\left.c_{1}\right), c_{3}$ ) and Remark 6.4, the bifunction $f_{T}$ given by (6.4) verifies the assumptions $\left(H_{1}\right)$ and $\left(H_{2}\right)$. In addition, with the global upper sign continuity of $T$, in view of assertion (i) of [7, Lemma 8.10], $f_{T}$ also satisfies the upper sign property assumption required in (ii) of Lemma 3.1. Moreover, for all $x \in C, f_{T}(x, x)=0$. Then $f_{T}$ trivially fulfils $\left(\mathbb{H}_{1}\right)$. At the meantime, condition $c_{4}$ ) ensures that $h_{2}$ ) holds with $f=f_{T}$. Finally, the quasiconvexity property of (ii) of Lemma 3.1 comes immediately from the convexity of $f_{T}$ in $y$. Then, all of the needed assumptions of Proposition 6.1 hold. So, the required conclusion is derived from this result immediately.

We are now ready to state our main result of sensitivity analysis for parametric quasi-equilibrium problem $\mathrm{QEP}\left(f, K_{\lambda}().\right)$ by making use of the following extension of Lim's Lemma ([29]).

Theorem 6.1. Let $X$ be a metric space, and let $T_{1}: X \rightrightarrows X$ and $T_{2}: X \rightrightarrows X$. Suppose that both $T_{1}$ and $T_{2}$ are Lipschitz continuous on $X$ with the same Lipschitz constant $\lambda \in[0,1)$. Then, for every $\varepsilon>0$, the set of $\varepsilon$-approximate fixed points of $T_{i}, i=1,2$, is nonempty, i.e., $\varepsilon$-Fix $\left(T_{i}\right) \neq \emptyset$, and moreover

$$
h\left(\varepsilon-\operatorname{Fix}\left(T_{1}\right), \varepsilon-\operatorname{Fix}\left(T_{2}\right)\right) \leqslant \frac{\varepsilon}{1-\lambda}+\frac{1}{1-\lambda} \sup _{x \in X} h\left(T_{1}(x), T_{2}(x)\right) .
$$

Proof From [30, Theorem 2.1.] (see also [31, Theorem 15]), we conclude the desired conclusion immediately.

Notation: For every $\lambda \in \Lambda$ and $\varepsilon>0$, we write $\varepsilon-\operatorname{SQ}(\lambda):=\varepsilon-\operatorname{SQ}\left(f, K_{\lambda}().\right)$ to stand for the set of $\varepsilon$-approximate solutions to $\operatorname{QEP}\left(f, K_{\lambda}().\right)$ according to Definition 4.1.

Theorem 6.2. Assume that conditions of Proposition 6.1 hold. Then, for every $\lambda \in \bar{V}$, and for every $\varepsilon>0$, the set of $\varepsilon$-approximate solutions to problem $\mathrm{QEP}\left(f, K_{\lambda}().\right)$ is nonempty, and moreover, for all $\lambda, \lambda^{\prime} \in \bar{V}$,

$$
h\left(\varepsilon-\operatorname{SQ}(\lambda), \varepsilon-\operatorname{SQ}\left(\lambda^{\prime}\right)\right) \leqslant \frac{1}{1-k}\left(\varepsilon+k\left\|\lambda-\lambda^{\prime}\right\|\right),
$$

where $k=\frac{2 R L}{m}$.

Proof Let $\varepsilon>0$. Using Proposition 6.1, the variational selection $S_{\lambda}$ of $\operatorname{QEP}\left(f, K_{\lambda}().\right)$ is a contracting map in $u$ with constant $k=\frac{2 R L}{m}<1$. Then, from [32, Theorem 1.2] or [30, Theorem 
2.1.], we have that $\varepsilon-\operatorname{Fix}\left(S_{\lambda}\right)$ is nonempty for every $\lambda \in \bar{V}$. Therefore, from Theorem 6.1 , it follows that

$$
\begin{aligned}
h\left(\varepsilon-\operatorname{Fix}\left(S_{\lambda}\right), \varepsilon-\operatorname{Fix}\left(S_{\lambda^{\prime}}\right)\right) & \leqslant \frac{\varepsilon}{1-k}+\frac{k}{1-k} \sup _{x \in X} h\left(S_{\lambda}(x), S_{\lambda^{\prime}}(x)\right) \\
& \leqslant \frac{\varepsilon}{1-k}+\frac{k}{1-k}\left\|\lambda-\lambda^{\prime}\right\| .
\end{aligned}
$$

From Lemma 4.1, for $\omega \in\left\{\lambda, \lambda^{\prime}\right\}$, we have

$$
\varepsilon-\operatorname{Fix}\left(S_{\omega}\right)=\varepsilon-\mathrm{SQ}(\omega) .
$$

Clearly, from (6.10) and the previous inequality, we conclude the required estimate in (6.9). This completes the proof.

Now, turn our attention to (QVI) and derive the following quantitative stability, where we denote by $\varepsilon$-S $\mathrm{QVI}(\lambda)$ the set of $\varepsilon$-approximate solutions to $\mathrm{QVI}\left(T, K_{\lambda}().\right)$ in the sense of Definition 5.1, for every $\lambda \in \bar{V}$ and $\varepsilon>0$.

Theorem 6.3. Assume that conditions of Corollary 6.1 hold. Then, for every $\lambda \in \bar{V}$, and for every $\varepsilon>0$, the set of $\varepsilon$-approximate solutions to problem $\mathrm{QVI}\left(T, K_{\lambda}().\right)$ is nonempty, and moreover, for all $\lambda, \lambda^{\prime} \in \bar{V}$,

$$
h\left(\varepsilon-\mathrm{S}_{\mathrm{QVI}}(\lambda), \varepsilon-\mathrm{S}_{\mathrm{QVI}}\left(\lambda^{\prime}\right)\right) \leqslant \frac{1}{1-k}\left(\varepsilon+k\left\|\lambda-\lambda^{\prime}\right\|\right)
$$

where $k=\frac{2 R L}{m}$.

Proof It suffices to apply Theorem 6.2 by taking $f=f_{T}$, where $f_{T}$ is the bifunction associated with the operator $T$ given by (6.4) above.

We next give similar sharp estimates for exact solutions to quasi-equilibrium problems and quasi-variational inequalities.

Theorem 6.4. Assume that either $C$ is compact or $X$ is complete, and the conditions of Theorem 6.2 hold. Then, the set of exact solutions to problem $\operatorname{QEP}\left(f, K_{\lambda}().\right)$ is nonempty, and moreover, for all $\lambda, \lambda^{\prime} \in \bar{V}$,

$$
h\left(\operatorname{SQ}(\lambda), \operatorname{SQ}\left(\lambda^{\prime}\right)\right) \leqslant \frac{1}{1-k}\left\|\lambda-\lambda^{\prime}\right\|
$$

where $k=\frac{2 R L}{m}<1$.

Proof Let $\lambda \in \bar{V}$. If $C$ is compact, then from [7, case (d) Theorem 7.9], QEP $\left(f, K_{\lambda}().\right)$ admits at least a solution, which means that the corresponding set of solutions is nonempty, i.e., $\mathrm{SQ}(\lambda) \neq \emptyset$. Now, from Proposition 6.1, one has that map $S_{\lambda}$ is a contraction. It follows from [31, e) Proposition 4] that $\operatorname{Fix}\left(S_{\lambda}\right)$ is closed. Moreover, from [31, e) Proposition 4], and for every $\varepsilon>0, \varepsilon-\mathrm{SQ}(\lambda)$ is closed. A further interesting property is that, for every sequence of positive real numbers decreasing to 0 , say $\varepsilon_{n} \searrow 0$, in view of [31, g) Proposition 4], $\left(\varepsilon_{n}-\mathrm{SQ}(\lambda)\right)_{n}$ converges to $\operatorname{SQ}(\lambda)$ in the sense of Painlevé-Kuratowski. This yields the convergence of this sequence in the Pompeiu-Hausdorff sense by the similar arguments to [33, Theorem 3A.4], i.e.,

$$
\lim _{n \rightarrow 0} h\left(\varepsilon_{n}-\mathrm{SQ}(\lambda), \mathrm{SQ}(\lambda)\right)=0 .
$$


On the other hand, for any other $\lambda^{\prime} \in \bar{V}$ with the triangle inequality of the Hausdorff distance, we are able to write, for every integer $n$,

$$
\begin{aligned}
h\left(\mathrm{SQ}(\lambda), \mathrm{SQ}\left(\lambda^{\prime}\right)\right) \leqslant & h\left(\mathrm{SQ}(\lambda), \varepsilon_{n}-\mathrm{SQ}(\lambda)\right)+h\left(\varepsilon_{n}-\mathrm{SQ}(\lambda), \varepsilon_{n}-\mathrm{SQ}\left(\lambda^{\prime}\right)\right) \\
& +h\left(\mathrm{SQ}\left(\lambda^{\prime}\right), \varepsilon_{n}-\mathrm{SQ}\left(\lambda^{\prime}\right)\right) .
\end{aligned}
$$

But, according to (6.9) of Theorem 6.2, we have

$$
h\left(\varepsilon_{n}-\mathrm{SQ}(\lambda), \varepsilon_{n}-\mathrm{SQ}\left(\lambda^{\prime}\right)\right) \leqslant \frac{1}{1-k}\left(\varepsilon_{n}+\left\|\lambda-\lambda^{\prime}\right\|\right)
$$

which implies that

$$
\begin{aligned}
h\left(\mathrm{SQ}(\lambda), \mathrm{SQ}\left(\lambda^{\prime}\right)\right) \leqslant & h\left(\mathrm{SQ}(\lambda), \varepsilon_{n}-\mathrm{SQ}(\lambda)\right)+\frac{1}{1-k}\left(\varepsilon_{n}+\left\|\lambda-\lambda^{\prime}\right\|\right) \\
& +h\left(\mathrm{SQ}\left(\lambda^{\prime}\right), \varepsilon_{n}-\mathrm{SQ}\left(\lambda^{\prime}\right)\right)
\end{aligned}
$$

Therefore, by taking into account the symmetry role of $\lambda$ and $\lambda^{\prime}$, and (6.13), the limit over $n$ in the previous inequality leads to

$$
h\left(\mathrm{SQ}(\lambda), \operatorname{SQ}\left(\lambda^{\prime}\right)\right) \leqslant \frac{1}{1-k}\left\|\lambda-\lambda^{\prime}\right\|,
$$

which is exactly the required estimate in (6.12). If $X$ is complete, then we conclude from Nadler's Theorem [34] that $S_{\lambda}$ admits a fixed point as a contracting map, which is in turn an exact solution to $\operatorname{QEP}\left(f, K_{\lambda}().\right)$. In this case, the estimate (6.12) follows from Lim's Lemma [29]. This completes the proof immediately.

For problem (QVI), we are able to obtain a similar conclusion to Theorem 6.4. Tut, for simplicity, we restrict ourselves to the compactness case for which we have another alternative to justify the existence of exact solutions without appeal to results of [7] or Nadler's contraction principle. We employ a convergence scheme of mixed approximate solutions in the sense of Definition 5.4. In this way, we state the following theorem.

Theorem 6.5. Assume that $C$ is compact, $T$ is Lipschitz continuous with constant $\mu \leqslant 1$, and the conditions of Corollary 6.1 hold. Then, for every $\lambda \in \bar{V}$, the set of exact solutions to problem $\mathrm{QVI}\left(T, K_{\lambda}().\right)$, say $\mathrm{S}_{\mathrm{QVI}}(\lambda)$, is nonempty, and moreover, for all $\lambda, \lambda^{\prime} \in \bar{V}$,

$$
h\left(\mathrm{~S}_{\mathrm{QVI}}(\lambda), \mathrm{S}_{\mathrm{QVI}}\left(\lambda^{\prime}\right)\right) \leqslant \frac{1}{1-k}\left\|\lambda-\lambda^{\prime}\right\|
$$

where $k$ is the constant given in Theorem 6.3.

Proof Let $\lambda \in \bar{V}$. From Corollary 6.1, it follows that $S_{\lambda}$ is a single-valued contraction with constant $k$ as defined above. Furthermore, by Theorem 6.3 , for every $\varepsilon>0, \varepsilon-\mathrm{S}_{\mathrm{QVI}}(\lambda)$ is nonempty. This means that, for every $\varepsilon>0$, problem $\mathrm{QVI}\left(T, K_{\lambda}().\right)$ admits an $\varepsilon$-approximate solution (in the sense of Definition 5.1). Thus, by Proposition 5.1, QVI $\left(T, K_{\lambda}().\right)$ admits a sequence of mixed $\varepsilon$-approximate solutions (in the sense of Definition 5.4) denoted by $\left(\bar{x}_{\varepsilon}\right)_{\varepsilon}$, that is, for every $\varepsilon>0$, there exists $\bar{x}_{\varepsilon} \in \varepsilon$-Fix $(K)$, and there exists $x_{\varepsilon}^{\star} \in T\left(\bar{x}_{\varepsilon}\right)$ such that

$$
\left\langle x_{\varepsilon}^{\star}, y-\bar{x}_{\varepsilon}\right\rangle \geqslant-\varepsilon-\varepsilon\left(\left\|x_{\varepsilon}^{\star}\right\|+\left\|y-\bar{x}_{\varepsilon}\right\|\right), \quad \forall y \in K\left(\bar{x}_{\varepsilon}\right) .
$$


Now, observe that the condition $c_{4}$ ) (of Corollary 6.1) implies the following condition $c_{6}$ ) of [25, Theorem 3.7]: For all $x, y \in C$, for all $x_{n} \rightarrow x$, and for all $y_{n} \rightarrow y$,

$$
\liminf _{n \rightarrow+\infty} \sup _{x_{n}^{\star} \in T\left(x_{n}\right)}\left\langle x_{n}^{\star}, y_{n}-x_{n}\right\rangle \geqslant 0 \Longrightarrow \sup _{x^{\star} \in T(x)}\left\langle x^{\star}, y-x\right\rangle \geqslant 0 .
$$

Hence, given that $T$ is a compact-valued map (since $C$ is compact), with the Sion's minimax Theorem as in [25, Theorem 3.7], we are able to claim that $\left(\bar{x}_{\varepsilon}\right)_{\varepsilon}$ converges to an exact solution to $\mathrm{QVI}\left(T, K_{\lambda}().\right)$, i.e., $\mathrm{S}_{\mathrm{QVI}}(\lambda)$ is nonempty. Finally, with the aid of Theorem 6.3 , the estimate required in (6.15) can be handled similarly to (6.12) of Theorem 6.4. This completes the proof.

Remark 6.7. Observe that if we consider, in Theorem 6.5, that operator $T$ is a continuous and strongly monotone-like single-valued map, then we obtain a partial improvement of the result in [35, Theorem 2] with respect to perturbations arising in feasible flows of traffic networks problems. Indeed, in [35, Theorem 2], with a parametric set of constraints (i.e., flows), it has been considered that those flows are norm-bounded from below (see [35, Lemma 2]), which is a restriction if such a traffic model assumes that flows are without a minimal value with respect to the norm. In our case, there is no further assumption on the constraints besides the Lipschitz behavior and common usual assumptions.

Remark 6.8. Despite the similarity of the schemes of the previous work [18] with the one of this paper, the extension of quantitative stability from quasi-optimization problems to general quasi-equilibrium ones is justified by the following:

- The main results of [18] are essentially based on the strong quasi-convexity property of the objective function. This condition may fail for some interesting classes of quasi-variational inequalities, for, e.g., the case in $\mathrm{QVI}(T, K()$.$) when T$ is single-valued.

- The results obtained in this paper cannot cover straightforwardly the mathematical programs studied in [18] due to the loss of information on strong monotonicity conditions in these programs. The results related to perturbation on objective functions in [18] are not, at least easily, extendable to quasi-equilibrium (resp. quasi-variational inequalities) problems with perturbed bifunctions (resp. set-valued operators) since the assumptions are different, which makes the difference at the level of computation of the bounds in the suggested estimates. This is why we restrict here the perturbation to constraints.

- The proposed extension here leads to devising new concepts of approximate solutions to quasi-variational inequalities that converge under appropriate regularity conditions to exact solutions.

- Following the Remark 6.6 above, and with the help of the new results in [27] on equilibrium problems, it is possible to get variants of our results in Theorem 6.2 and Theorem 6.4 if we replace the strong monotonicity-like property on the bivariate function $f$ by the strong quasi-convexity-like condition of $f$ in the second variable. With such an alternative in the main assumption, and with respect to perturbed constraints, we can consider this paper as a non-trivial generalization and unification of the previous results.

- The same remark as the previous one may be done on quasi-variational inequalities. In fact, our Theorems 6.3 and 6.5 may be adapted to strongly quasimonotone-like operators to weaken the strong monotonicity condition. This possibility would give a perspective to 
cover quasi-optimization and quasi-convex programming in the framework of QVI problems by considering the normal operator adjusted to sub-levels of the objective function, see [15].

\section{Acknowledgements}

The authors thank the editors and the anonymous referees for very useful comments and suggestions which helped them to improve this paper

\section{REFERENCES}

[1] E. Blum, W. Oettli, From optimization and variational inequalities to equilibrium, Math. Student 63 (1994), 123-145.

[2] M. Castellani, M. Guili, Existence of quasiequilibria in metric vector spaces, J. Math. Anal. Appl. 484 (2020), $1-13$.

[3] M. Castellani, M. Guili, M. Pappalardo, A Ky Fan minimax inequality for quasiequilibria on finite dimensional spaces, J. Optim. Theory Appl. 179 (2018), 53-64.

[4] D. Aussel, J. Cotrina, A. Iusem, An existence result for quasi-equilibrium problems, J. Convex Anal. 24 (2017), 55-66.

[5] J. Cotrina, J. Zúñiga, Quasi-equilibrium problems with non-self constraint map, J. Glob. Optim. 75 (2019), $177-197$.

[6] J. Cotrina, J. Zúñiga, A note on quasi-equilibrium problems, Oper. Res. Lett. 46 (2018), 138-140.

[7] M. Ait Mansour, R-.A. Elakri, M. Laghdir, Equilibrium and quasi-equilibrium problems under $\phi$ quasimonotonicity and $\phi$-quasiconvexity: Existence and stability, Minimax Theory Appl. 2 (2017), 175-229.

[8] P.Q. Khanh, N.H Quan, Versions of the Weiertrass theorem for bifunctions and solution existence in optimization, SIAM J. Optim. 29 (2019), 1502-1523.

[9] G. Bigi, M. Castellani, M. Pappalardo, M. Passacantando, Nonlinear Programming Techniques for Equilibria, Springer, 2019.

[10] G. Kassay, V. Radulescu, Equilibrium Problems and Applications, Elsevier, 2018.

[11] A. Göpfert, H. Riahi, C. Tammer, C. Zălinescu, Variational Methods in Partially Ordered Spaces, Springer, New York 2003.

[12] N.D. Yen, Lipschitz continuity of solutions of variational inequalities with a parametric polyhedral constraint, Math. Oper. Res. 20 (1995), 695-707.

[13] M. Ait Mansour, H. Riahi, On the eigenvalues problem for hemivariational inequalities: Existence and stability, J. Convex Anal. 9 (2002), 309-325.

[14] M. Ait Mansour, H. Riahi, Sensitivity analysis for abstract equilibrium problems, J. Math Anal. Appl. 306 (2005), 684-691.

[15] M. Ait Mansour, D. Aussel, Quasimonotone variational inequalities and quasiconvex programming: Quantitative stability, Pacific J. Optim. 3 (2006), 611-626.

[16] J. Gwinner, Stability of monotone variational inequalities with various applications, In: F. Giannessi, A. Maugeri, (eds) Variational Inequalities and Network Equilibrium Problems, pp. 123-142, Boston, Springer, 1995. https://doi.org/10.1007/978-1-4899-1358-6_10

[17] M. Ait Mansour, D. Aussel, Quasimonotone variational inequalities and quasiconvex programming: qualitative stability, J. Convex Anal. 15 (2008), 459-472.

[18] M. Ait Mansour, M.A. Bahraoui, A. El Bekkali, Sharp estimates for approximate and exact solutions to quasi-optimization problems, Optimization, https://doi.org/10.1080/02331934.2021.1873986

[19] M. Bianchi, R. Pini, Coercivity conditions for equilibrium problems, J. Optim. Theory Appl. 124 (2005), 79-92.

[20] M. Castellani, M. Giuli, Refinements of existence results for relaxed quasimonotone equilibrium problems, J. Glob. Optim. 57 (2013), 1213-1227.

[21] O. Chadli, Z. Chbani, H. Riahi, Equilibrium problems with generalized monotone bifunction and application to variational inequalities, J. Optim. Theory Appl. 105 (2000), 209-333. 
[22] F. Flores-Bazán, Existence theorems for generalized noncoercive equilibrium problems: the quasiconvex case, SIAM J. Optim. 11 (2000), 675-690.

[23] A. N. Iusem, G. Kassay, W. Sosa, On certain conditions for the existence of solutions of equilibrium problems, Math. Program. 116 (2009), 259-273.

[24] W. Oettli, A remark on vector-valued equilibria and generalized monotonicity, Acta Math. Vietnamica 22 (1997), 213-221.

[25] M. Ait Mansour, J. Lahrache, N. Ziane, $\alpha$-strong approximate solutions to quasi-variational inequalities, Le Matematiche 73 (2018), 115-125.

[26] B. S. Mordukhovich, Variational Analysis and Generalized Differentiation I: Basic Theory, Springer-Verlag, 2006.

[27] M. Ait Mansour, R.A. Elakri, M. Laghdir, Quantitative stability for quasimonotone equilibrium problems under relaxed upper-sign properties: Application to quasiconvex programming, Appl. Anal. Optim. 3 (2019), 307-331.

[28] N. Hadjisavvas, Continuity and maximality properties of pseudomonotone operators, J. Convex Anal. 10 (2003), 465-475.

[29] T. C. Lim, On fixed-point stability for set-valued contractive mappings with applications to generalized differential equations, J. Math. Anal. Appl. 110 (1985), 436-441.

[30] M. Ait Mansour, M.A. Bahraoui, A. El Bekkali, A global approximate contraction mapping principle in non-complete metric spaces, J. Nonlinear Var. Anal. 4 (2020), 153-157.

[31] M. Ait Mansour, M.A. Bahraoui, A. El Bekkali, Metric regularity and Lyusternik-Graves Theorem via approximate fixed points of set-valued maps in noncomplete metric spaces, Set-Valued Var. Anal. (2020). https://doi.org/10.1007/s11228-020-00553-1

[32] M. Ait Mansour, M.A. Bahraoui, A. El Bekkali, Approximate fixed points via completion, Le Matematiche 74 (2019), 411-415.

[33] A.L. Dontchev, R.T. Rockafellar, Implicit Functions and Solution Mappings, Springer, New York, 2014.

[34] S. B. Nadler, Multivalued contraction mappings. Pacific J. Math. 30 (1969), 475-488.

[35] M. Ait Mansour, L. Scrimali, Hölder continuity of solutions to elastic traffic network, J. Glob. Optim. 40 (2008), 175-184. 